\title{
DER STAND DER AFRIKANISCHEN \\ VERFASSUNGSGESETZGEBUNG \\ UND DIE NEUEN VERFASSUNGSSAMMLUNGEN
}

\author{
Von HaRTwig Rogge
}

I.

Die Anzahl der Sammlungen ausschließlich oder unter anderem afrikanischer Verfassungen hat sich in den vergangenen Jahren vermehrt. $\mathrm{Zu}$ den bekannten Editionen von Lavroff-Peiser ${ }^{1}$ und Peaslee $^{2}$ sind eine Ausgabe afrikanischer Verfassungstexte hin italienischer Sprache von Foderaro, eine vom Sekretariat des Asian-African Legal Consultative Committee bearbeitete englischsprachige Ausgabe, zwei Loseblattsammlungen und das Vorhaben des Corpus Constitutionnel hinzugekommen.

Salvatore Foderaro (Le Costituzioni Africane, Rom 1969) beschränkt sich auf die reine Textwiedergabe. Die wesentlich aktuellere Sammlung des Asian-African Legal Consultative Committee (Constitutions of African States, Neu Delhi 1972) versieht die Texte demgegenüber mit einer einführenden Vorbemerkung nebst einer Bibliographie. Für beide Sammlungen sei einschränkend vermerkt, daß sie nicht in jeder Hinsicht zuverlässig sind und schon zum Zeitpunkt der Herausgabe zum Teil überholt waren. Als geschlossene Sammlungen besitzen sie wie die Editionen von Lavroff-Peiser und Peaslee ferner den Nachteil, daß sie angesichts der wechselvollen afrikanischen Verfassungsentwicklung rasch veralten. Gleichwohl stellt die Sammlung des Committee eine hilfreiche Informationsquelle und nützliche Arbeitsgrundlage dar.

Der ebenfalls englischsprachigen Loseblattsammlung von Blaustein-Flanz (Constitutions of the Countries of the World, New York $1971 \mathrm{ff}$.$) und den „Verfassungs-$ texten" (Beilage zu Verfassung und Recht in Übersee, Hamburg 1972 ff.) ist ihre Aktualität gemeinsam. Hinsichtlich ihres Umfangs und ihrer Aufmachung können die noch in den Anfängen begriffenen „Verfassungstexte“ mit Blaustein-Flanz allerdings nicht verglichen werden. Beide Ausgaben versehen die Texte mit einer Vorbemerkung und einer Bibliographie, wobei die im Stile einer Verfassungschronologie verfaßten Vorbemerkungen bei Blaustein-Flanz teilweise an Qualität gewonnen haben.

Eine Verfassungsedition, die in ihrer Sorgfalt und Gründlichkeit jedem Vergleich standhält, ist das mit Unterstützung der UNESCO in Angriff genommene Corpus Constitutionnel (Leiden 1968 ff.). Es ist bisher leider nur bis zum Buchstaben B vorgedrungen.

II.

Die nachfolgende Übersicht ist bemüht, den neuesten Stand (April 1974) der afrikanischen Verfassungsgesetzgebung zu erfassen. Soweit die zur Zeit maßgeblichen Texte zum Abdruck gekommen sind, wird in der Klammer auf die hier aufgeführ-

\footnotetext{
1 Lavroff-Peiser, Les Constitutions Africaines, L'Afrique Noire Francophone et Madagascar, Paris 1961, Etats Anglophones, Paris 1964.

2 Amos J. Peaslee, Constitutions of Nations, Vol. I Africa, 3. Auflage, Den Haag 1965.
} 
ten Sammlungen verwiesen. Das Kürzel „Constitutions“ bezeichnet die Ausgabe des Asian-African Legal Consultative Committee.

Ägypten: Verfassung von 1971 (Constitutions, Blaustein-Flanz, Verfassungstexte) (Statut der Einheitspartei [Verfassungstexte])

(FAR: Verfassung von 1971 [Foderaro])

Äquatorial Guinea: Verfassung (von 1968) 1971 suspendiert

Äthiopien: Revidierte Verfassung von 1955 (Peaslee, Foderaro, Constitutions, Blaustein-Flanz)

Algerien: Verfassung von 1963 (Peaslee, Foderaro, Constitutions, Blaustein-Flanz, Corpus Constitutionnel), „Kleine Verfassung“ von 1965 (Corpus Constitutionnel) Botswana: Verfassung von 1966 (Foderaro, Constitutions, Blaustein-Flanz, Corpus Constitutionnel), Verfassungsänderungen 1969 (Blaustein-Flanz, Corpus Constitutionnel), 1970 (Blaustein-Flanz), 1972

Burundi: Verfassung (von 1962) 1966 suspendiert, Verfassungsgesetz und -dekrete 1966, 1968, 1971 (Statut der Einheitspartei [Blaustein-Flanz])

Dahome: Verfassung (von 1970) 1972 suspendiert, Verfassungsproklamation, -dekrete und -ordonnanzen 1972, 1973

Elfenbeinküste: Verfassung von 1960 (Lavroff-Peiser) i. d. F. von 1963 (Peaslee, Foderaro, Constitutions, Blaustein-Flanz)

Gabun: Verfassung von 1961 (Lavroff-Peiser, Peaslee) i. d. F. von 1963 (Foderaro), i. d. F. von 1967 (Constitutions), Verfassungsänderungen 1968, 1969.

Gambia: Verfassung von 1970 (Blaustein-Flanz)

Ghana: Verfassung (von 1969) 1972 aufgehoben, Verfassungsdekrete 1972 (Blaustein-Flanz)

Guinea: Verfassung von 1958 (Lavroff-Peiser, Peaslee, Foderaro, Constitutions, Blaustein-Flanz), Verfassungsänderung 1963

Kamerun: Verfassung von 1972 (Blaustein-Flanz, Verfassungstexte)

Kenya: Verfassung von 1969 (Neufassung) (Constitutions, Blaustein-Flanz)

Kongo: Verfassung von 1973

Lesotho: Interimsverfassung von 1973

Liberia: Verfassung von 1847 i. d. F. von 1955 (Lavroff-Peiser, Peaslee, Foderaro, Constitutions, Blaustein-Flanz)

Libyen: Interimsverfassung von 1969 (Constitutions)

Madagaskar: Provisorische Verfassung von 1972, Verfassungsordonnanzen 1973

Malawi: Verfassung von 1966 (Foderaro) i. d. F. von 1966 (Constitutions, Blaustein-Flanz), Verfassungsänderungen 1969-1973

Mali: Provisorische Verfassung von 1968 (Constitutions)

Marokko: Verfassung von 1968 (Constitutions)

Mauretanien: Verfassung von 1961 (Lavroff-Peiser, Peaslee, Constitutions) i. d. F. von 1966 (Foderaro), i. d. F. von 1971 (Blaustein-Flanz)

Mauritius: Verfassung von 1968 (Foderaro, Constitutions, Blaustein-Flanz), Verfassungsänderung 1973

Niger: Verfassung (von 1960) 1974 suspendiert

Nigeria: Verfassung von 1963 (Lavroff-Peiser, Peaslee, Foderaro, Constitutions), Verfassungsänderungen 1966, 1967, 1970 (z. T. Constitutions, z. T. Blaustein-Flanz) Obervolta: Verfassung (von 1970) 1974 suspendiert, Verfassungsproklamation 1974 Ruanda: Verfassung von 1962 (Peaslee, Foderaro, Constitutions, Blaustein-Flanz), Verfassungsänderungen 1973 (provisorische Neuordnung), 1974 
Sambia: Verfassung von 1973 (Verfassungstexte)

Senegal: Verfassung von 1963 (Peaslee, Foderaro) i. d. F. von 1967 (Constitutions), Verfassungsänderungen 1968, 1970

Sierra Leone: Verfassung von 1971 (Blaustein-Flanz), Verfassungsänderungen 1971, 1972 (Blaustein-Flanz)

Somalia: Verfassung (von 1960) 1969 aufgehoben

Sudan: Verfassung von 1973

Südafrika: Verfassung von 1961 (Lavroff-Peiser, Peaslee) i. d. F. von 1963 (Foderaro), i. d. F. von 1967 (Blaustein-Flanz), Verfassungsänderung 1971

Swasiland: Verfassung von 1968 (Foderaro, Blaustein-Flanz), Verfassungsproklamation 1973

Tansania: Verfassung von 1965 (Foderaro, Constitutions) i. d. F. von 1972 (Blaustein-Flanz)

Togo: Verfassung (von 1963) 1967 suspendiert

Tschad: Verfassung von 1962 (Peaslee) i. d. F. von 1965 (Constitutions), i. d. F. von 1967 (Foderaro)

Tunesien: Verfassung von 1959 (Peaslee, Constitutions) i. d. F. von 1965 (Foderaro), i. d. F. von 1966 (Blaustein-Flanz), Verfassungsänderungen 1969, 1970

Uganda: Verfassung von 1967 (Constitutions), Verfassungsänderungen 1970—1972 (z. T. Constitutions, Blaustein-Flanz)

ZAR: Verfassungsgesetz von 1966 (Blaustein-Flanz)

Zaïre: Verfassung von 1967 (Foderaro, Blaustein-Flanz), Verfassungsänderungen 1970, 1971 (Verfassungstexte), 1972, 1973 (Statut der Einheitspartei [Verfassungstexte]) 